CI: 0.49-0.99]). None of the biological factors were associated with high levels of PA.

Conclusion Social and behavioural factors appear to be the most influential factors driving PA levels. The results should be used to guide the development of educational campaigns and behaviour change intervention to encourage younger women to be active and to target those with lower education attainment and of lower socioeconomic status.

\section{P14 ASSOCIATION OF SLEEP DURATION AND SLEEP QUALITY WITH THE LOSS OF FUNCTIONING AMONG AUSTRALIAN PEOPLE}

\begin{abstract}
${ }^{1,2}{ }^{2}$ L Lallukka*, ${ }^{3,4,9} \mathrm{BS}$ Sivertsen , ${ }^{1}$ EK Kronholm, ${ }^{5} \mathrm{YSB}$ Bin, ${ }^{6,7}$ SØ Øverland, ${ }^{8} \mathrm{NG}$ Glozier, ${ }^{1} \mathrm{JHH}$ Halonen. ${ }^{1}$ Research and Service Centre for Work Organisations, Finnish Institute of Occupational Health, Helsinki and Turku, Finland; '2Department of Public Health, University of Helsinki, Helsinki, Helsinki; ${ }^{3}$ Department of Health Promotion, Norwegian Institute of Public Health, Bergen, Norway; ${ }^{4}$ The Regional Centre for Child and Youth Mental Health and Child Welfare, Uni Research Health, Bergen, Norway; ${ }^{5}$ Central Clinical School, Sydney Medical School, University of Sydney, Sydney, Australia; ${ }^{6}$ Norwegian Institute of Public Health, Bergen, Norway; ' Department of Psychosocial Science, University of Bergen, Bergen, Bergen; ${ }^{8}$ Brain and Mind Centre, University of Sydney, Sydney, Australia; ${ }^{9}$ Department of Research and Innovation, Helse Fonna HF, Haugesund, Norway
\end{abstract}

\subsection{6/jech-2017-SSMAbstracts.116}

Background While short and long sleep duration and poor sleep quality have individually been linked to work disability and ill-health, their joint impact on functioning has received less attention. The aim was to examine the joint associations of sleep quantity and quality with physical, emotional, and social functioning, while considering the contribution of key potential confounders.

Methods Data from the nationally representative Household, Income and Labour Dynamics in Australia (HILDA) Study, collected in 2013, were used $(n=14557)$. Poor sleep quality (yes/ no) was examined in combination with normal (6-8 hours), short $(<6)$ or long $(>8)$ sleep. Short form SF-12 questionnaire was used to examine functioning. Covariates included sociodemographic factors, social connexions (meeting with friends), health behaviours, obesity, pain, and mental and physical illnesses. Logistic regression analysis was used (odd ratios, OR and their 95\% confidence intervals, 95\% CI).

Results After adjusting for sex and age, short, normal and long sleep duration in combination with poor quality were all associated with poorer physical, emotional and social functioning. The associations were relatively similar for physical and emotional functioning, and strongest for social functioning (OR 3.36, 95\% CI 2.94-3.84 for normal sleep with poor sleep quality, OR 4.56, 95\% CI 4.02-5.17 for short sleep with poor sleep quality and OR 7.83, CI 95\% 6.09-10.09 for long sleep with poor sleep quality). Also people with good sleep quality but short or long sleep had poorer functioning, but the associations were weaker for all forms of functioning (ORs ranging 1.28-1.57 after adjusting for sex and age). Pain and comorbid illness attenuated all of the associations the most, while the contributions of the other covariates were relatively minor. However, the associations remained after full adjustments for people with poor sleep quality and short, normal or long sleep, and for people with good sleep quality but long sleep duration.
Conclusion The results highlight that in particular poor sleep quality is likely to have the most severe consequences for daytime functioning in the general population, and that there is little difference between short and normal sleepers in the presence of poor quality sleep.

\section{P15 INEQUALITIES IN ALCOHOL-RELATED MORTALITY: AN ELECTRONIC COHORT STUDY OF INTERNAL MIGRATION IN WALES}

A Gartner*, D Farewell, G Greene, L Trefan, S Paranjothy, D Fone. Division of Population Medicine, School of Medicine, Cardiff University

\subsection{6/jech-2017-SSMAbstracts. 117}

Introduction Alcohol-related deaths show a strong socioeconomic gradient. Selective migration, when individuals in poorer health move to more deprived areas, or vice versa, is associated with widening health inequality. However, it is not known whether recent internal migration contributes to the observed health inequality in alcohol-related deaths. This study aims to investigate whether selective migration of individuals is associated with the alcohol-related mortality gradient.

Methods We constructed a record-linked electronic cohort of the total population of Wales registered with a GP on 01/01/ 2006 ( $n=3$ 136881) with six-years' follow-up to 31/12/2011 in the SAIL Databank at Farr@Swansea. The Welsh Demographic dataset provided information on age, sex, and the lower super output area (LSOA) $(n=1896)$ of residence at each of the 24 quarters, used to code migration. To each LSOA code we linked tenths of the Welsh Index of Multiple Deprivation 2008 and the ONS Rural-Urban definition. To code alcohol-related mortality ( $n=2224$ of 184247 deaths) using the standard ONS definition we linked the demographic dataset to the ONS mortality files.

We estimated hazard ratios (HR) with 95\% confidence intervals $(95 \% \mathrm{CI})$ using Cox regression for the risk of alcohol-related death for deprivation fifths, adjusting for agegroup, sex and rural-urban status. The first model estimated HRs allowing for changing deprivation fifths linked to each move and the second model fixed the deprivation fifth at the start of the study period. A third model considered deprivation change between high (the five most deprived tenths) and low deprivation (the five least deprived tenths).

Results We found 495 subjects moved between LSOAs prior to an alcohol-related death. Of these, the majority (260) moved between high deprivation LSOAs and 75 moved between low deprivation LSOAs. Slightly more subjects moved from areas of low to high deprivation (98) than the opposite (62).

In model 1 subjects living in the most deprived fifth had a higher risk of alcohol-related death (HR 3.20; 95\% CI 2.77 to 3.71) compared to subjects in the least deprived fifth. In model 2 the risk was little different (HR 2.96; 95\% CI 2.56 to 3.42). Formal statistical adjustment for recent migration had little effect on the inequality estimates. In model 3, there was an elevated risk of alcohol-related death for moving from low to high deprivation.

Conclusion Internal migration within Wales over a six-year period does not explain the observed inequality in alcoholrelated mortality. 